\title{
DOES LONELINESS HAVE NATIONALITY? CULTURAL AND INDIVIDUAL DIFFERENCES IN LONELINESS AMONG AMERICAN AND POLISH YOUNG ADULTS
}

\author{
Chris Segrin ${ }^{1}$, Katarzyna Adamczyk ${ }^{2}$, Corey A. Pavlich ${ }^{3}$, Malgorzata Osowiecka ${ }^{4}$, \\ Radosław Trepanowski ${ }^{2}, \&$ Agata Celejewska ${ }^{2}$ \\ ${ }^{1}$ Department of Communication, University of Arizona (USA) \\ ${ }^{2}$ Adam Mickiewicz University in Poznan (Poland) \\ ${ }^{3}$ School of Communication, Cleveland State University (USA) \\ ${ }^{4}$ University of Social Sciences and Humanities in Warsaw (Poland)
}

\begin{abstract}
The current study was intended to determine the possible differences in social and emotional (i.e., family and romantic) loneliness a function of nationality and relationship status. Although the social construction of romantic relationships in Poland and the US has changed over recent years, the Polish culture with its stronger traditionalist value system may still place a higher premium on having a romantic or marital partner compared to the US. The family unit is also a more central and core element of one's social network in Poland compared to the US. Associations between loneliness and romantic partner status were tested in 431 US and 395 Polish participants (mean age $=22.92$ ) who completed the Social and Emotional Loneliness Scale for Adults - Short Form (SELSA-S; DiTommaso, Brannen, \& Best, 2004). The analyses demonstrated that Polish respondents reported higher loneliness than US respondents, Wilks' $\lambda=0.89, F(3,769)=30.78$, $p<.001$ and single individuals reported higher loneliness than partnered individuals, Wilks' $\lambda=0.60$, $F(3,769)=169.26, p<.001$. There were also significant nationality and relationship status interactions in the domain of family loneliness, $F(1,771)=6.47, p<.01$, and in the domain of romantic loneliness, $F(1,771)=71.82, p<.001$. More specifically, single Polish participants reported higher family loneliness than Polish partnered participants. American participants reported the exact same level of family loneliness, regardless of their relationship status. In turn, the lack of a romantic partner was more strongly associated with increased romantic loneliness for Polish than for American participants. Although all participants reported increased loneliness when they were without a partner, this effect was particularly exaggerated among Polish participants. At the same time, Polish participants seem to benefit more (in terms of decreased romantic loneliness) than Americans do as a function of having a romantic partner.
\end{abstract}

Keywords: Loneliness, relationship status, young adults, Poland, USA.

\section{Introduction}

In the research literature loneliness is conceptualized as a multifaceted or domain-specific phenomenon. The first researcher who described loneliness as a multidimensional experience was Weiss (1973) who proposed a distinction between social loneliness as a result of an inadequate access to social networks and emotional loneliness perceived as a lack of close or intimate relationships which are characteristic of ties with a romantic partner, parent, or child. Emotional loneliness is primarily related to "the absence of a partner, that is, with the absence of an exclusive, close, and intimate tie" (Dykstra \& Fokkema, 2007, p. 9). In turn, social loneliness is related to a perceived deficiency in social networks, or a lack of social relations or social activities (Russell, Cutrona, Rose, \& Yurko 1984; Weiss 1973). Furthermore, on the basis of Weiss' (1973) distinction between the experience of social isolation (social loneliness) and emotional isolation (emotional loneliness), DiTommaso and Spinner $(1993,1997)$ noted that emotional loneliness appeared to be comprised of two domains, that is, family emotional loneliness and romantic emotional loneliness.

Prior studies showed that the lack of a meaningful relationship with a partner or a spouse in emerging and young adulthood may be an important factor for the experience of loneliness (e.g., Russell, Cutrona, McRae, \& Gomez, 2012). At the same time, it is important to emphasize that in most prior studies loneliness was conceptualized as a unitary state. This unidimensional approach to loneliness recognizes 
loneliness as basically having the same form in different social contexts (e.g. Russell, Peplau, \& Cutrona, 1980). Research by DiTommaso and Spinner (1993) however indicated that involvement in a romantic relationship is associated with lower romantic loneliness, but to a weak degree with family and social loneliness. Furthermore, in a Polish study single individuals scored higher on romantic and family loneliness than did partnered individuals, and no differences emerged between single and partnered individuals in the domain of social loneliness (Adamczyk, 2016).

Alongside the discussion concerning the link between relationship status and loneliness it is also important to consider the fact that various cultures and societies may hold diverse attitudes and beliefs about marriage and possessing a partner (Gubernskaya, 2010). For instance, Poland has unique qualities derived from a traditional notion of life paths in adulthood, which includes heterosexual marriage and having children, relatively low rate of divorces, and less approval for alternative family and marital forms. In contrast, the American society is characterized by a higher level of divorces, and seems to be more approving towards alternative forms of marital and family life such as singlehood, marital non-cohabitation, and cohabitation without marriage. As a result, singlehood in Poland might be related to lower life satisfaction outcomes in comparison to singlehood in the United States of America, where singlehood might not be so detrimental for these outcomes.

\section{Objectives}

The primary aim of the present investigation was to determine the possible differences in social and emotional (i.e., family and romantic) loneliness a function of nationality and relationship status. In addition, we were also interested to determine if there is an interaction between nationality and relationship status such that differences between single and partnered people in terms of loneliness across the two nationalities. In regard to the linkage between relationship status and social and emotional (i.e., family and romantic) loneliness we expected that:

H1. Single individuals in Poland and in the United States would report higher romantic loneliness than partnered individuals.

H2. There will be an interaction between nationality and relationship status that Polish single individuals would report higher romantic loneliness than American single individuals.

\section{Method}

\subsection{Participants and procedure}

The study was conducted on a sample of 431 US and 395 Polish participants $(M=22.92$, $S D=4.24$ ) Women represented $67 \%$ of the sample and men represented $30 \%$, with $3 \%$ missing this information. There were 461 participants $(56 \%)$ who reported being in a romantic relationship at the time of the assessment, while 335 participants $(41 \%)$ were not, and $30(4 \%)$ were missing this information.

To recruit Polish and American participants, the authors distributed questionnaires in the form of online survey using a computer program 'Qualtrics' available at many American universities. Qualtrics allows users to view and complete the survey at their own pace on their computer, while storing all of the responses in single data file.

\subsection{Materials}

The questionnaire presented to participants was comprised of the following instruments:

Demographic Questionnaire. A series of demographic questions was asked to obtain general descriptive information about participants' background such as their age, sex, education level, and current relationship status.

The Social and Emotional Loneliness Scale for Adults - Short Form (SELSA-S; DiTommaso et al., 2004) (Polish adaptation - Adamczyk \& DiTommaso 2014). The SELSA-S is a multidimensional measure of loneliness that consists of 15 items rated on a 7-point Likert-type scale, ranging from 1 (strongly disagree) to 7 (strongly agree). It was designed to measure emotional (romantic and family) and social loneliness. Each subscale consists of five statements about feelings of loneliness within the past year. The family loneliness subscale assesses feelings toward family relationships. The social loneliness subscale measures feelings toward being part of a social group. The romantic loneliness subscale measures the degree to which participants feel they have significant others in their lives. Mean scores are calculated for each subscale, and higher SELSA-S scores indicate higher levels of loneliness in the particular domain. The SELSA-S's three subscales have high internal reliability, with Cronbach's alpha coefficients ranging from .87 to .90 , and have been shown to be a valid measure of loneliness (DiTommaso et al., 2004). 


\section{Results}

The analyses demonstrated that Polish respondents reported higher loneliness than US respondents, Wilks' $\lambda=0.89, F(3,769)=30.78, p<.001$ and single individuals reported higher loneliness than partnered individuals, Wilks' $\lambda=0.60, F(3,769)=169.26, p<.001$. There were also significant nationality and relationship status interactions in the domain of family loneliness, $F(1,771)=6.47, p<.01$, and in the domain of romantic loneliness, $F(1,771)=71.82, p<.001$. More specifically, single Polish participants reported higher family loneliness than Polish partnered participants. American participants reported the exact same level of family loneliness, regardless of their relationship status. In turn, the lack of a romantic partner was more strongly associated with increased romantic loneliness for Polish than for American participants.

\section{Discussion}

The objective of the present study was to investigate whether potential differences in social and emotional (i.e., family and romantic) loneliness exists as a function of Polish and American nationality and relationship status among young adults. The findings revealed that Polish individuals experienced higher loneliness in comparison to their American counterparts and that single individuals reported higher loneliness than partnered individuals in both countries (H1). The analyses also revealed a significant interaction between nationality and relationship status in the domain of family and romantic loneliness. To be precise, single individuals in Poland reported higher family loneliness than Polish partnered participants, whereas American participants reported the same level of family loneliness, regardless of their relationship status. Moreover, we determined that single status was related to increased romantic loneliness in Poland in comparison to American participants and this pattern of results confirmed $\mathrm{H} 2$.

The observed interaction between nationality and relationship status in the domain of romantic loneliness as a result of the cultural and social specificity of Poland and the United States of America suggests that the differentiation in importance and evaluation of marriage and family [termed the Ideology of Marriage and Family by DePaulo and Morris (2005)] translate into the experience of increased loneliness associated with singlehood. Therefore, we may conclude that in cultures and societies where possessing a partner/spouse and marriage are highly valued (Poland) life satisfaction outcomes (i.e., loneliness) are lower among single people compared to their peers in cultures and societies that place a lower premium on possessing a partner or marriage (USA).

There are several limitations of this study. First, the correlational nature of the study precludes any causal inferences concerning the observed associations. Second, the age of the sample, even though representing a unique developmental state, precludes any generalizations to individuals in middle and late adulthood. For example, the consequences of singlehood and urgency for finding a life partner would presumably be very different among middle aged adults. Despite these limitations, the present findings underscore the importance of understanding how cultural values and norms may influence people's experience of possessing or not a partner and the possible consequences of relationship status.

\section{Acknowledgments}

The presented project proposal is financed by a grant from the Polish Scientific National Centre as part of the research project "A Longitudinal investigation of mental and physical health of Polish and American young adults” (UMO-2014/13/B/HS6/01382).

\section{References}

Adamczyk, K. (2016). An investigation of loneliness and perceived social support among single and partnered young adults. Current Psychology, 35(4), 674-689. doi: 10.1007/s12144-015-9337-7

Adamczyk, K., \& DiTommaso, E. (2014). Psychometric properties of the Polish version of the Social and Emotional Loneliness Scale for Adults (SELSA-S). Psychological Topics, 23(3), 327-341. UDK - 159.923.33.072-057.875(438)

DePaulo, B., Morris, W. (2005). Singles in society and science. Psychological Inquiry, 16(2), 57-83. doi: 10.1207/s15327965pli162\&3_01 
DiTommaso, E., Brannen, C., \& Best, L. A. (2004). Measurement and validity characteristics of the short version of the Social and Emotional Loneliness Scale for Adults. Educational and Psychological Measurement, 64, 99-119. doi: 10.1177/0013164403258450

DiTommaso, E., \& Spinner, B. (1993). The development and initial validation of the Social and Emotional Loneliness Scale for Adults (SELSA). Personality and Individual Differences, 14, 127-134. doi: 10.1016/0191-8869(93)90182-3

DiTommaso, E., \& Spinner, B. (1997). Social and emotional loneliness: A re-examination of Weiss' typology of loneliness. Personality and Individual Differences, 22, 417-427. doi: 10.1016/S0191-8869(96)00204-8

Dykstra, P.A., \& Fokkema, T. (2007). Social and emotional loneliness among divorced and married men and women: comparing the deficit and cognitive perspectives. Basic and Applied Social Psychology, 29(1), 1-12. doi: 10.1080/01973530701330843

Gubernskaya, Z. (2010). Changing attitudes toward marriage and children in six countries. Sociological Perspectives, 53(2), 179-200. doi: 10.1525/sop.2010.53.2.179

Russell, D. W., Cutrona, C., McRae, C., \& Gomez, M. (2012). Is loneliness the same as being alone? The Journal of Psychology, 146(1-2), 7-22. doi: 10.1080/00223980.2011.589414

Russell, D., Cutrona, C.E., Rose, J., \& Yurko, K. (1984). Social and emotional loneliness: An exploration of Weiss's typology of loneliness. Journal of Personality and Social Psychology, 46(6), 1313-1321. doi: 10.1037/0022-3514.46.6.1313

Russell, D., Peplau, L. A., \& Cutrona, C. E. (1980). The revised UCLA Loneliness Scale: Concurrent and discriminant validity evidence. Journal of Personality and Social Psychology, 39(3), 472-480.

Weiss, S. R. (1973). Loneliness: The experience of emotional and social isolations. London, England: The MIT Press. 